\title{
Re-visiting the detection of porcine cysticercosis based on full carcass dissections of naturally Taenia solium infected pigs
}

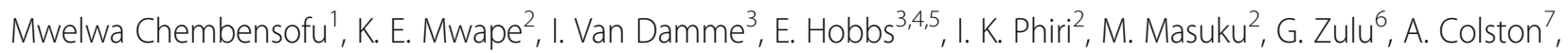
A. L. Willingham ${ }^{4}$, B. Devleesschauwer ${ }^{8}$, A. Van Hull, A. Chota ${ }^{1}$, N. Speybroeck' ${ }^{9}$ D. Berkvens ${ }^{5}$, P. Dorny ${ }^{3,5}$ and S. Gabriël $\left.\right|^{*}$

\begin{abstract}
Background: Taenia solium is a neglected zoonotic parasite. The performances of existing tools for the diagnosis of porcine cysticercosis need further assessment, and their shortcomings call for alternatives. The objective of this study was to evaluate the performance of tongue palpation and circulating antigen detection for the detection of porcine cysticercosis in naturally infected pigs of slaughter age compared to full carcass dissections (considered the gold standard). Additionally, alternative postmortem dissection procedures were investigated. A total of 68 rural pigs of slaughter age randomly selected in the Eastern Province of Zambia were dissected. Dissections were conducted on full carcasses (or half carcass in case cysticerci were already detected in the first half), including all the organs. Total cysticercus counts, location and stages were recorded and collected cysticerci were identified morphologically and molecularly. All sera were analysed with the B158/B60 antigen detecting ELISA (Ag-ELISA).

Results: Key findings were the high occurrence of T. solium infected pigs (56\%) and the presence of T. solium cysticerci in the livers of $26 \%$ of infected animals. More than half of the infected carcasses contained viable cysticerci. Seven carcasses had T. hydatigena cysticerci (10\%), out of which five carcasses were co-infected with T. hydatigena and T. solium; two carcasses (3\%) had only T. hydatigena cysticerci. Compared to full carcass dissection, the specificity of the Ag-ELISA to detect infected carcasses was estimated at $67 \%$, the sensitivity at $68 \%$, increasing to $90 \%$ and $100 \%$ for the detection of carcasses with one or more viable cysticerci, and more than 10 viable cysts, respectively. Tongue palpation only detected $10 \%$ of the cases, half carcass dissection $84 \%$.

Selective dissection of the diaphragm, tongue and heart or masseters can be considered, with an estimated sensitivity of $71 \%$, increasing to $86 \%$ in carcasses with more than 10 cysticerci.

Conclusions: Depending on the aim of the diagnosis, a combination of Ag-ELISA and selective dissection, including investigating the presence of T. hydatigena, can be considered. Full carcass dissection should include the dissection of the liver, kidneys, spleen and lungs, and results should be interpreted carefully, as small cysticerci can easily be overlooked.
\end{abstract}

Keywords: Taenia solium, Cysticercosis, Pig carcass, Dissection, Liver, Antigen ELISA, Tongue palpation

\footnotetext{
* Correspondence: sarah.gabriel@ugent.be

${ }^{3}$ Faculty of Veterinary Medicine, Ghent University, Salisburylaan 133, 9820

Merelbeke, Belgium

Full list of author information is available at the end of the article
} 


\section{Background}

Taenia solium, also known as the pork tapeworm, is a zoonotic parasite which is endemic in developing countries of Africa, Asia and Latin America where pigs are raised as a food source and are kept under free range conditions [1]. The tapeworm causes two disease conditions in humans, taeniosis (intestinal tapeworm infection) and cysticercosis (metacestode larval stage infection). Infection of the central nervous system with cysticerci leads to a condition known as neurocysticercosis (NCC), which is the most prevalent helminthic infection of the nervous system and a leading cause of acquired epilepsy worldwide [2]. Porcine cysticercosis is acquired when pigs get access to human faeces containing T. solium ova. Cysticerci are primarily located in muscle tissues and the brain [1]. This condition may lead to carcass condemnations and hence extensive economic losses which can be significant $(50-60 \%$ of the carcass value) in some countries [3, 4].

Zambia is endemic for T. solium infection with prevalences of, porcine cysticercosis ranging from 14\% (AgELISA) to 64\% (Bayesian estimation) [5, 6]; human cysticercosis between 5.8-15\% (Ag-ELISA); and human taeniosis between 6.3-12\% (Copro-Ag ELISA) [7, 8]. Recent results indicated over $50 \%$ of cases of acquired epilepsy (late onset) are due to NCC [9]. There is therefore an urgent need to control and possibly eliminate the parasite in Zambia.

A number of control strategies, such as human and/or pig treatment, pig vaccination, health education (reviewed by [10]) have been evaluated by observing the changes in prevalence of porcine cysticercosis through diagnostic procedures like tongue palpations, routine meat inspection, serological techniques and carcass dissections. Performances of these tests have been estimated (using a Bayesian approach, confirmed using dissection results of 65 pigs) at $21 \%$ sensitivity (Se) and $100 \%$ specificity (Sp) for tongue palpation and $22 \%$ Se and $100 \%$ Sp for meat inspection $[6,11]$. Serological techniques, detecting circulating antigens and specific antibodies are frequently used, although test performances are a matter of debate and need further assessment. The monoclonal antibody-based circulating antigen detecting ELISAs have been reported to have a Se of $65-93 \%$ and a Sp of $70-100 \%$ in detecting porcine cysticercosis in naturally infected pigs [6, 12]; for the detection of specific antibodies the best performances are obtained with the enzyme-linked immunoelectrotransfer blot (EITB), with a sensitivity of $78-100 \%$ and a specificity of $43-100 \%[13,14]$. Carcass dissection (in $5 \mathrm{~mm}$ slices) is generally considered the gold standard diagnostic method in assessing infection status. It can describe the infection intensity, and stage, size and distribution of cysticerci in different organs $[6,11]$. The major disadvantages of full carcass dissection are, the costs involved in purchasing of pigs, the labor, the time needed, and the need for trained personnel to conduct the dissections. To reduce labor and time, many researchers aim for half carcass dissections, though the performance of this method as a diagnostic tool needs to be assessed thoroughly. An additional disadvantage of carcass dissection is that the animals are removed from the study population, which can impact on the results in follow up studies of control interventions. As such, relatively few dissections have been done on naturally infected pigs in sub-Saharan Africa $[6,15,16]$.

Clearly there is a need to assess the performances of currently available diagnostic tools for porcine cysticercosis, using full carcass dissection as a reference method, and to define valid alternatives to full carcass dissections.

The objective of this study was to evaluate the performance of tongue palpation and circulating antigen detection for the detection of porcine cysticercosis in naturally infected pigs of slaughter age via comparison with full carcass dissections. Additionally, alternative postmortem dissection procedures, such as half carcass dissection and specific organ/muscle group dissections, were investigated for their effectiveness to detect infected animals.

\section{Methods \\ Study site}

The CYSTISTOP Project is currently implementing measures for elimination/control of Taenia solium cysticercosis in Nyembe and Mtandaza rural communities of Katete and Sinda Districts, in the Eastern Province of Zambia. A baseline study was conducted between October and November 2015 in the study areas to establish the baseline prevalence of porcine cysticercosis through full carcass dissection (half carcass dissection if cysticerci were already identified in the first half of the carcass). The two districts were selected following the high prevalence of porcine and human cysticercosis/taeniosis reported by previous studies $[5,8,9,11]$. Other factors considered were the accessibility of the areas and willingness to participate of the village representatives (village headmen) and health/veterinary officers.

\section{Sample size and pig selection}

The sample size of pigs selected for postmortem examinations was calculated for the intervention study (CYSTISTOP, see above), and was based on $80 \%$ power to detect an effect of intervention using a one-sided likelihood ratio test at the $5 \%$ significance level with an assumption for prevalence of $20 \%$ before interventions. Calculations of the sample size were done using SAS 9.3, with the TWOSAMPLEFREQ command in the PROC POWER procedure (sample size $=67$ ).

A list of pig households $(\mathrm{HH})$ in the study area was drawn in excel, from which a random list of $\mathrm{HH}$ was 
generated (random function in excel) and pigs were purchased (one pig per $\mathrm{HH}$ ). All pigs of slaughter weight and age from the selected $\mathrm{HH}$, and to which the owner agreed to sell, were given arbitrary numbers. Random sampling was done by casting a dice and the number at which the dice settled was noted, the pig with that corresponding number was selected and purchased.

\section{Tongue palpation and euthanasia}

Tongue examination/palpation was performed before slaughter by placing each pig in left lateral recumbence, where it was firmly restrained by three people. The mouth was opened by a wooden rod and the tongue pulled gently with a mutton cloth $[6,11]$. Pigs were considered positive for cysticercosis if cyst-like nodules were either seen or felt [14] on any part of the tongue. Animals were slaughtered by a captive bolt gun, and thereafter exsanguinated.

\section{Blood collection, sera separation and storage}

Immediately after exsanguination, blood was collected from either the jugular or the cranial vena cava veins in $50 \mathrm{ml}$ falcon tubes. To maximize the amount of serum collected, the blood was placed in the refrigerator at $4{ }^{\circ} \mathrm{C}$ overnight. The following day, the blood was centrifuged at $3000 \times \mathrm{rpm}$ for $15 \mathrm{~min}$. Sera were separated and aliquoted in duplicate cryovials and stored at $-20{ }^{\circ} \mathrm{C}$ until use.

\section{Carcass dissection and cysticercus counting}

Carcasses and heads were skinned and the muscles separated from the bones. Muscle tissues/organs were excised from one carcass half of each pig, together with the complete head (eyes and brain) heart, tongue, neck, diaphragm, psoas muscles, spleen, kidneys, lungs and liver. Slices of maximum $0.5 \mathrm{~cm}$ thickness were made and cysticerci were enumerated. If cysticerci were identified in the half carcass, the cysticercus counts for the muscles of the half carcass were doubled and added to the counts of the complete head, heart, tongue, neck, diaphragm, psoas muscles, spleen, kidneys, lungs, liver, eyes and brain to obtain total carcass counts. If no cysticerci were found in the first half of the carcass, then the other half of the carcass was dissected (= full carcass dissection). When the number of cysticerci was too high to accurately count, 100 cysticerci per muscle/organ part was assumed.

Cysticerci were classified as viable if they had a translucent fluid with a visible whitish protoscolex; degenerated if they had a collapsed and viscous damaged cysticercus wall and absence of the cystic fluid; or calcified if they were non-cystic and with yellowish caseous masses $[11,16]$. These were observed macroscopically and recorded on pig dissection spread sheets. Taenia hydatigena cysts were also looked for in the peritoneal cavity, on intestinal surfaces and in the visceral organs (livers, lungs, heart, kidney, diaphragm and omentum).

\section{Cysticercus viability test}

A few cysticerci (1-4, depending on availability) were collected from all positive carcasses, washed in phosphate buffered saline (PBS) and put onto Petri dishes containing a 1:1 mixture of pig bile and normal saline, as described by [14]. They were incubated at room temperature for $2 \mathrm{~h}$ to allow for complete evagination of cysticercus scolices.

\section{Laboratory analysis of serum samples}

The B158/B60 sandwich enzyme-linked immunosorbent assay (Ag-ELISA) was performed on sera to detect circulating antigens as described by Dorny et al. [6]. The status of the test samples was determined by comparing their optical densities to those of 8 negative control sera (from Zambian pigs) at a probability $(P)<0.001$ [17].

\section{PCR-restriction fragment length polymorphism (PCR-RFLP) analysis}

A number of cysticerci (at least 1) detected in the muscles were collected from all dissection-positive carcasses for confirmation by PCR-restriction fragment length polymorphism (PCR-RFLP) as described by Dermauw et al. [18]. Cysticerci detected in organs (e.g. liver, spleen and lungs) were also collected separately for each organ for molecular confirmation by PCR-RFLP.

\section{Data management and analysis}

Data were entered into EpiData twice and statistical analyses were performed in STATA/IC 14.1 (StataCorp LP, College Station, TX, USA). Pigs were considered $T$. solium positive if at least one cysticercus was detected and confirmed as $T$. solium. Univariate logistic regressions were performed to evaluate differences between age (6-12 months $v s \geq 13$ months), region, and sex. The sensitivity of using one (multiple) organ(s) to detect $T$. solium (an organ was considered positive when at least one $T$. solium cysticercus was detected) was estimated relative to whole carcass dissection results as criterion standard. Similarly, the sensitivity and specificity of the Ag-ELISA were estimated relative to whole carcass dissection results, considering a carcass as positive when (i) at least one $T$. solium cysticercus was detected; (ii) at least ten T. solium cysticerci were detected; (iii) at least one viable $T$. solium cysticercus was detected; (iv) at least ten viable $T$. solium cysticerci were detected. When determining the sensitivity and specificity of tests, exact binomial 95\% confidence intervals were calculated according to Clopper \& Pearson [19]. To evaluate the relation between the number of cysticerci and the Ag-ELISA ratio, the animals were grouped according to the total 
number of cysticerci: group 0 (between 1 and 10 cysticerci); group 1 (between 11 and 99 cysticerci) and group 2 ( $\geq 100$ cysticerci). The Ag-ELISA ratios between each of the groups were compared using Wilcoxon rank-sum tests and the trend across the ordered groups was evaluated according to Cuzick [20]. The same was done to evaluate the relation between the number of viable cysticerci and Ag-ELISA ratio.

\section{Results}

\section{Overall dissection results}

A total of 68 pigs were selected and purchased for dissections (Nyembe, $n=37$; Mtandaza, $n=31$ ). All pigs dissected were local breeds indigenous to the Eastern Province of Zambia.

Cysticerci were detected by dissection in a total of 38 carcasses (56\%), of which 32 were detected during the dissection of the first half of the carcass. An additional six cases were detected only during the dissection of the second half of the remaining 36 carcasses (full carcass dissection). As such, half carcass dissection would have picked up 84\% (95\% CI: 69-94\%) of the infected carcasses. Taenia solium cysticerci were found in 4/68 (6\%) pigs by tongue palpation. Tongue palpation detected 4 out of the 38 (11\%; 95\% CI: 2.9-25\%) dissection positives.

No significant difference was determined in infection status between male (66\% infected, 21/32) and female pigs (49\% infected, $17 / 35$, the sex of one animal was not noted $)(\mathrm{OR}=2.0 ; 95 \% \mathrm{CI}: 0.7-5.4, P=0.162)$. In Nyembe and Mtandaza, $46 \%$ and $68 \%$ of pigs were $T$. solium positive, respectively, though the difference between both areas was not significant $(\mathrm{OR}=0.4$; $95 \%$ CI: $0.1-1.1, P=0.074$ ).

In the two age group categories; $6-12$ months of age and $\geq 13$ months, the infection occurrences were determined at 59\% (24/41) and 52\% (14/27), respectively, no significant differences were observed $(\mathrm{OR}=1.3$; $95 \% \mathrm{CI}$ : $0.5-3.5, P=0.587$ ).

Taenia hydatigena cysticerci were identified in seven carcasses, representing 10\% (7/68) of the total carcasses dissected, and all of these cysticerci were viable. Of the seven $T$. hydatigena positives, five carcasses were coinfected with $T$. hydatigena and T. solium. The other two carcasses (3\%) had only T. hydatigena cysticerci. A total of $10 \mathrm{~T}$. hydatigena cysticerci were observed in those seven carcasses.

\section{Infection intensity}

The number of cysticerci per carcass dissected ranged from 1 to $>10,000$. A high proportion of infected carcasses $(76 \%)$ had low to moderate infection levels $(42 \%$ with $\leq 10$ cysticerci and $34 \%$ with 11-50 cysticerci). High infection levels of $>100$ cysticerci were observed in $21 \%$ of infected carcasses, of which one carcass had $>10,000$ cysticerci (exact number of cysticerci was impossible to count) (Table 1$)$. In one carcass, very small $(2 \mathrm{~mm})$ cysticerci were detected. The majority of these small cysticerci had intact walls, semitransparent membrane and transparent cysticercus fluids. Several of these small cysticerci from this pig were subjected to the viability test as well and all of them evaginated, thus demonstrating their viability. The viable cysticerci collected from the carcasses and subjected to viability tests also evaginated within two hours.

\section{Distribution and classification of $T$. solium cysticerci}

In $24 \%$ of the infected carcasses only viable cysticerci were detected, in $11 \%$ only degenerated cysticerci and in $16 \%$ only calcified cysticerci. In 13 (34\%) of the infected carcasses, cysticerci of various developmental stages were observed in the same carcass, and all three stages were present in seven carcasses.

The distribution and number of T. solium cysticerci in different locations are shown in Table 2. The carcass with more than 10,000 cysticerci has not been included in Table 2, as no exact counts were conducted. A total of 3382 cysticerci were detected in the different tissues and organs of the $37 \mathrm{~T}$. solium infected carcasses. Of the 3382 cysticerci detected, 2925 (86.5\%) were viable, 127 (3.8\%) were degenerated and 330 (9.8\%) were calcified. In 4 carcasses, cysticerci were localized only in one organ/tissue in each case (pig 29: liver; pig 31: heart; pig 33: hind leg; pig 39: tongue). The brain recorded the lowest number of cysticerci (107), however most of them were viable $(102 / 107 ; 95.3 \%)$. The liver recorded the lowest percentage of viable cysticerci $(110 / 151 ; 72.9 \%)$ and highest percentage of calcified cysticerci $(34 / 151$; $22.5 \%)$. The foreleg recorded the highest number of $T$. solium cysticerci (1003).

In this study, ten out of 38 infected carcasses had cysticerci in the liver (26\%; 95\% CI: 13-43\%), one carcass had a cysticercus in the spleen $(2.6 \%$; $95 \%$ CI: $0.6-14 \%)$ and two carcasses had cysticerci in the lungs (5.3\%; 95\% CI: 0.6-18\%).

In almost all the positive cases, $T$. solium cysticerci were detected in at least one or more predilection sites, except the one carcass that only had cysticerci in the liver. In carcasses infected with 1-10 cysticerci, most

Table 1 Taenia solium infection levels in infected carcasses

\begin{tabular}{ll}
\hline No. of cysticerci in carcass & No. of carcasses (\%) \\
\hline$\leq 10$ & $16(42)$ \\
$11-50$ & $13(34)$ \\
$51-99$ & $1(3)$ \\
$\geq 100$ & $7(18)$ \\
$>10,000$ & $1(3)$ \\
Total & $38(100)$ \\
\hline
\end{tabular}


Table 2 Distribution and stage of the total number of Taenia solium cysticerci detected in the different muscles/organs in 37 infected carcasses

\begin{tabular}{lllll}
\hline $\begin{array}{l}\text { Muscle/ } \\
\text { organ }\end{array}$ & \multicolumn{2}{l}{ Cysticercus stage } & $\begin{array}{l}\text { Total } \\
\text { cysticerci/ } \\
\text { organ (\%) }\end{array}$ \\
\cline { 2 - 4 } Viable & Degenerated & Calcified & $158(4.7)$ \\
\hline Masseter & 145 & 2 & 11 & $145(4.3)$ \\
Heart & 132 & 2 & 11 & $172(5.1)$ \\
Tongue & 153 & 1 & 18 & $197(5.8)$ \\
Psoas & 177 & 3 & 17 & $215(6.4)$ \\
Diaphragm & 187 & 5 & 23 & $107(3.2)$ \\
Brain & 102 & 0 & 5 & $1003(29.7)$ \\
Foreleg & 883 & 22 & 98 & $791(23.4)$ \\
Hind leg & 657 & 40 & 94 & $151(4.5)$ \\
Liver & 110 & 7 & 34 & $443(13.2)$ \\
Other & 379 & 45 & 19 & $3382(100)$ \\
Total (\%) & $2925(86)$ & $127(4)$ & $330(10)$ & \\
\hline
\end{tabular}

Note: The carcass with more than 10,000 viable cysticerci is not included in the table

carcasses had cysticerci in the foreleg. For infected carcasses with 11-99 cysts, most carcasses had cysticerci in the hind and foreleg (Table 3).

When examining the effectiveness of dissection of different organ/tissue combinations ('selective dissection') instead of full carcasses for the detection of $T$. solium cysticercosis in pigs, the two equally optimal combinations were dissection of diaphragm, tongue and heart; and diaphragm, tongue and masseter. These combinations would each detect 27 of the 38 infected carcasses (71\%; 95\% CI: 54-85\%) (Table 4). In infected carcasses with more than ten cysticerci, selectively dissecting only the diaphragm, tongue and masseters, or the combination diaphragm, heart and masseters, would have detected 19 of the 22 infected carcasses (86\%; 95\% CI: 65-97\%) (Table 5).

\section{Ag-ELISA}

Of the 68 dissected pigs, a total of 36/68 (53\%) tested positive for $T$. solium cysticerci circulating antigens by AgELISA. Of the four tongue positives, three tested positive on Ag-ELISA. Of the $38 \mathrm{~T}$. solium dissection positive carcasses, 26 serum samples tested positive $(68 \%$; 95\% CI: 51-82\%). From the 30 T. solium dissection negative carcasses, Ag-ELISA returned ten positives, of which two were infected with $T$. hydatigena (based on dissection).

Considering the full carcass dissections as gold standard, the sensitivity and specificity of the Ag-ELISA for the detection of porcine cysticercosis (all cysticercus stages) were determined at $68 \%$ (95\% CI: $51-82 \%)$ and 67\% (95\% CI: $47-83 \%$ ), respectively. The sensitivity increased to $91 \%$ (95\% CI: 71-99\%) for carcasses with ten or more cysticerci. The Ag-ELISA was originally developed specifically to detect viable cysticerci as only these release antigens in the circulation [21]. If only viable cysticerci are considered, the sensitivity of the Ag-ELISA to detect carcasses with viable cysticerci (1 or more) was estimated at 91\% (95\% CI: 71-99\%), and increased further to $100 \%$ (95\% CI: $75-100 \%)$ for carcasses with at least 10 viable cysticerci (Table 6).

Figures 1 and 2 present the Ag-ELISA ratios (OD/cutoff) in relation to the infection levels detected in the carcasses. In $T$. solium dissection positive carcasses, the Ag-ELISA ratios were higher in pigs with $\geq 100$ cysticerci $(n=8)$ than in pigs with $11-99$ cysticerci $(n=14$; $Z=2.525, P=0.012)$, and the latter group had higher ratios compared to pigs that had $\leq 10$ cysticerci $(n=16$; $Z=3.016, P=0.003)$. Similarly, pigs with $\geq 100$ viable cysticerci $(n=7)$ had higher ratios than pigs with 11-99

Table 3 Number of carcasses with Taenia solium cysticerci in the specified muscle/organ of infected carcasses according to the overall infection level of the pig

\begin{tabular}{|c|c|c|c|c|}
\hline \multirow[t]{2}{*}{ Muscle/organ } & \multicolumn{3}{|c|}{ Infection level (total number of T. solium cysticerci/carcass) } & \multirow{2}{*}{$\begin{array}{l}\text { Total } \\
(n=38)\end{array}$} \\
\hline & $\begin{array}{l}\text { 1-10 cysticerci } \\
(\mathrm{n}=16)^{\mathrm{a}}\end{array}$ & $\begin{array}{l}\text { 11-99 cysticerci } \\
(\mathrm{n}=14)^{\mathrm{a}}\end{array}$ & $\begin{array}{l}\geq 100 \text { cysticerci } \\
(n=8)^{\mathrm{a}}\end{array}$ & \\
\hline Masseter & 1 & 5 & 7 & 13 \\
\hline Heart & 2 & 4 & 8 & 14 \\
\hline Tongue & 4 & 6 & 7 & 17 \\
\hline Psoas & 0 & 2 & 8 & 10 \\
\hline Diaphragm & 5 & 5 & 7 & 17 \\
\hline Brain & 0 & 1 & 6 & 7 \\
\hline Foreleg & 8 & 11 & 8 & 27 \\
\hline Hind leg & 5 & 11 & 8 & 24 \\
\hline Liver & 5 & 2 & 3 & 10 \\
\hline Other organs/tissues & 5 & 9 & 8 & 22 \\
\hline
\end{tabular}


Table 4 Number of infected carcasses identified by 'selective dissection' of different organ/tissue combinations for the detection of $T$. solium cysticercosis in 38 confirmed infected pig carcasses

\begin{tabular}{llllll}
\hline & Heart & Tongue & Diaphragm & Masseter & Psoas \\
\hline Heart & 14 & - & - & - & - \\
Tongue & 21 & 17 & - & - & - \\
Diaphragm & 22 & 25 & 17 & - & - \\
Masseter & 19 & 21 & 21 & 13 & - \\
Psoas & 16 & 19 & 19 & 16 & 10 \\
Heart and tongue & na & na & 27 & 25 & 22 \\
Heart and diaphragm & na & 27 & na & 25 & 23 \\
Heart and masseter & na & 25 & 25 & na & 21 \\
Heart and psoas & na & 22 & 23 & 21 & na \\
Tongue and diaphragm & 27 & na & na & 27 & 25 \\
Tongue and masseter & 25 & na & 27 & na & 23 \\
Tongue and psoas & 22 & na & 25 & 23 & na \\
Diaphragm and masseter & 25 & 27 & na & na & 22 \\
Diaphragm and psoas & 23 & 25 & na & 22 & na \\
Masseter and psoas & 21 & 23 & 22 & na & na \\
\hline
\end{tabular}

Abbreviation: na not applicable

viable cysticerci $(n=6 ; Z=2.143, P=0.032)$, which had higher Ag-ELISA ratios than pigs with $\leq 10$ viable cysticerci $(n=9 ; Z=2.121, P=0.034)$. An overall significant increasing trend was found across the groups for both total and viable cysticerci $(Z=4.30$ and $Z=3.72$, $P<0.001)$. Ratios from the ten non-infected carcasses

Table 5 Number of infected carcasses identified by 'selective dissection' of different organ/tissue combinations for the detection of $T$. solium cysticercosis in 22 confirmed infected pig carcasses with more than ten cysts

\begin{tabular}{llllll}
\hline & Heart & Tongue & Diaphragm & Masseter & Psoas \\
\hline Heart & 12 & - & - & - & - \\
Tongue & 15 & 13 & - & - & - \\
Diaphragm & 16 & 17 & 12 & - & - \\
Masseter & 16 & 16 & 16 & 12 & - \\
Psoas & 14 & 15 & 14 & 15 & 10 \\
Heart and tongue & na & na & 18 & 18 & 16 \\
Heart and diaphragm & na & 18 & na & 19 & 17 \\
Heart and masseter & na & 18 & 19 & na & 18 \\
Heart and psoas & na & 16 & 17 & 18 & na \\
Tongue and diaphragm & 18 & na & na & 19 & 17 \\
Tongue and masseter & 18 & na & 19 & na & 18 \\
Tongue and psoas & 16 & na & 17 & 18 & na \\
Diaphragm and masseter & 19 & 19 & na & na & 17 \\
Diaphragm and psoas & 17 & 17 & na & 17 & na \\
Masseter and psoas & 18 & 18 & 17 & na & na \\
\hline
\end{tabular}

Abbreviation: na not applicable that tested positive by Ag-ELISA were generally low (1.10-3.98), except for two carcasses in which $T$. hydatigena cyst(s) were detected (ratio 27.02 and 40.19). T. hydatigena cyst(s) were detected in seven of the 68 dissected carcasses, of which six tested positive on $\mathrm{Ag}$ ELISA (ratios ranging between 2.64-43.13) and one tested negative (ratio 0.79).

\section{Molecular identification of cysticerci (PCR-RFLP)}

For all cysticerci morphologically identified as $T$. solium, one cysticerc collected from the muscles of each carcass (38) and at least one cysticerc collected from each organ per carcass (liver: 10; spleen: 1; lungs: 2) was analysed. All the analysed cysticerci from the $38 \mathrm{~T}$. solium dissection-positive carcasses (muscle tissues and other organs, including the very small cysts) were confirmed by PCR-RFLP as T. solium. All T. hydatigena cysts identified morphologically at dissection were confirmed by PCR-RFLP as T. hydatigena.

\section{Discussion}

This study recorded a high prevalence (56\%) of porcine cysticercosis in the pigs studied in the Eastern Province of Zambia, based on half and full carcass dissections of randomly selected pigs of slaughter age. This figure is in the same magnitude as the earlier $64 \%$ prevalence estimation of porcine cysticercosis in Zambia based on a Bayesian analysis of serological, tongue palpation and routine meat inspection results $[6,11]$.

The fact that $56 \%$ of the slaughter-aged pigs studied were infected with this zoonotic parasite despite the intervention methods employed by various stakeholders (e.g. measures to improve sanitation) is worrying for public health. This is of additional concern given that more than half of the infected carcasses (58\%) had viable cysticerci. The cysticerci present in the meat of the randomly selected pigs of slaughter age in this study would have potentially entered the food chain, with $87 \%$ of the cysticerci viable (infectious). In studies in Cameroon and Peru, 19.6 and $16.8 \%$ of the carcasses were infected based on (half) carcass dissection, respectively. Infection levels differ between these countries with $20 \%$ of the infected pigs in Cameroon having less than 50 cysticerci, while this was $50 \%$ in Peru [22]. In our study, most carcasses $(76 \%)$ had $1-50$ cysticerci.

Tongue palpation detected 4 of the 38 (11\%) dissection positives, confirming its low sensitivity for detecting porcine cysticercosis [6]. Indeed, in a previous study conducted in Zambia, tongue palpation detected $7.7 \%$ of infected pigs, while meat inspection detected $18.5 \%$ of infected pigs (infection status determined by carcass dissections) [11]. Half carcass dissection, often used as a substitute for full carcass dissection, would have detected $84 \%$ of the infected carcasses in our study, 
Table 6 Test performances of the Ag-ELISA according to the infection level and cyst stage in 38 confirmed infected pigs of slaughter age

\begin{tabular}{llll}
\hline Taenia solium infection level and stage & Ag-ELISA-positive & Ag-ELISA-negative & Sensitivity (95\% Cl) \\
\hline Carcasses with $\geq 1$ cysticercus $(n=38)$ & 26 & 12 & $68(51-83)$ \\
Carcasses with $\geq 10$ cysticerci $(n=22)$ & 20 & 2 & $91(71-99)$ \\
Carcasses with $\geq 1$ viable cysticercus $(n=22)$ & 20 & 2 & $91(71-99)$ \\
Carcasses with $\geq 10$ viable cysticerci $(n=13)$ & 13 & 0 & $100(75-100)$ \\
\hline
\end{tabular}

representing a certain loss in sensitivity (Se), while the workload still remains substantial. An alternative described by Lightowlers et al. [22] suggested dissecting only the tongue, heart and masticatory muscles, which, in their study including animals from Cameroon and Peru, led to a detection of $81 \%$ of the infected animals. In our study, dissection of these organs would have led to the detection of $66 \%$ of infected carcasses only, suggesting that the effectiveness of this approach might vary, and is likely dependent on infection intensity. Also, in the study of Lightowlers et al. [22], carcasses from animals from Cameroon were only half dissected, which, according to our results can lead to a non-detection of $16 \%$ of cases, and as such affect the 'gold standard'. Including the diaphragm, tongue and masseter led to a detection of $71 \%$ of cases in our study, increasing to $86 \%$ in carcasses infected with ten or more cysticerci. Of course, choice of organs/muscle groups that can be dissected will be influenced by other aspects such as size and price of the organ, ease of slicing and recognising the cysticerci, consumers' preferences amongst others. Whether a sensitivity of $71 \%$ for this 'selective dissection' is sufficient and a better option than the use of serological tools is debatable, and largely dependent on the aim of detection.

A similar sensitivity was observed for the Ag-ELISA, a much more user friendly and cheaper technique, which can be applied ante mortem and will as such not impact ongoing interventions. The main drawback is its low specificity (67\%), though this can be resolved by (selective) dissections of the positives. The latter should be completed by a thorough investigation for presence of $T$. hydatigena cysts. When the aim is to reduce the risk for public health, that is removal of infectious (viable) cysticerci from the food chain, the use of the Ag-ELISA would be more effective with sensitivities of $91 \%$ for carcasses with 1 or more viable cysticerci, and 100\% for carcasses with more than 10 cysticerci. Lack of sensitivity and specificity has been described for antibody detecting serological tools as well [13]. The routinely used EITB for detection of human (neuro)cysticercosis, fails to deliver the same performance in pigs, where sensitivities and specificities have been re-assessed at $89 \%$ and $43 \%$; respectively [12]. In a recent initiative, the Target

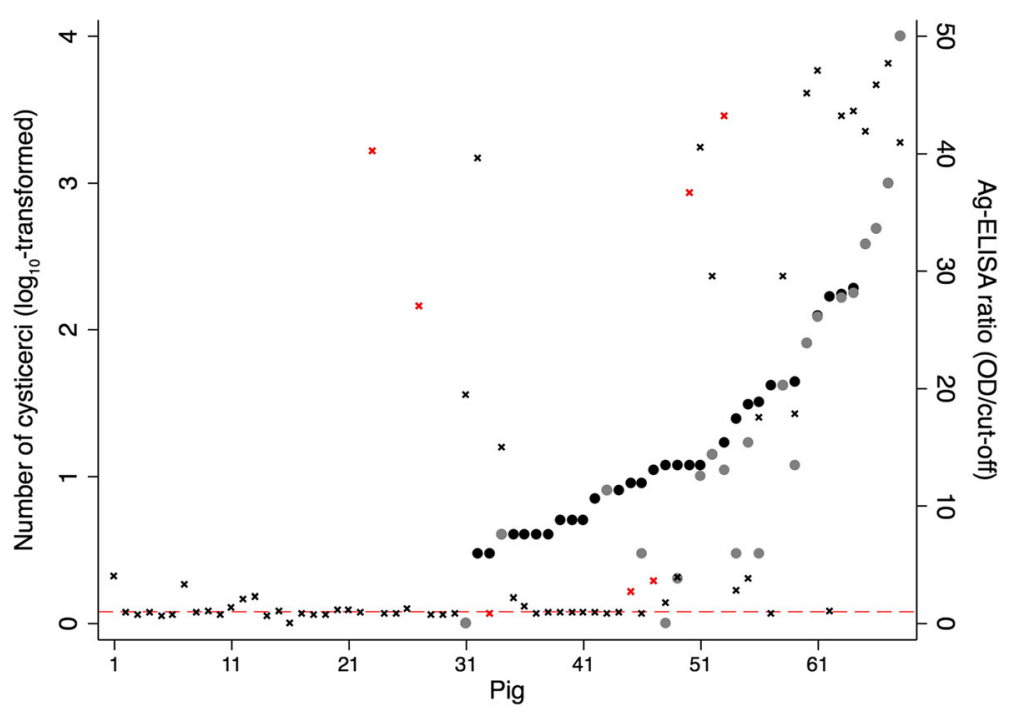

Fig. 1 Overview of the T. solium cysticercosis infection level (total number of (viable) cysticerci; $\log _{10}$-transformed; total number of cysticerci indicated with black circles and number of viable cysticerci with grey circles) and Ag-ELISA ratio (OD/cut-off; indicated with $\mathrm{x}$ ) per pig ( $n=68)$. Ag-ELISA ratios in black and red indicate $T$. hydatigena negative and positive animals, respectively. The dashed horizontal line indicates the cut-off value for a positive ELISA result, i.e. ratio $=1$ 


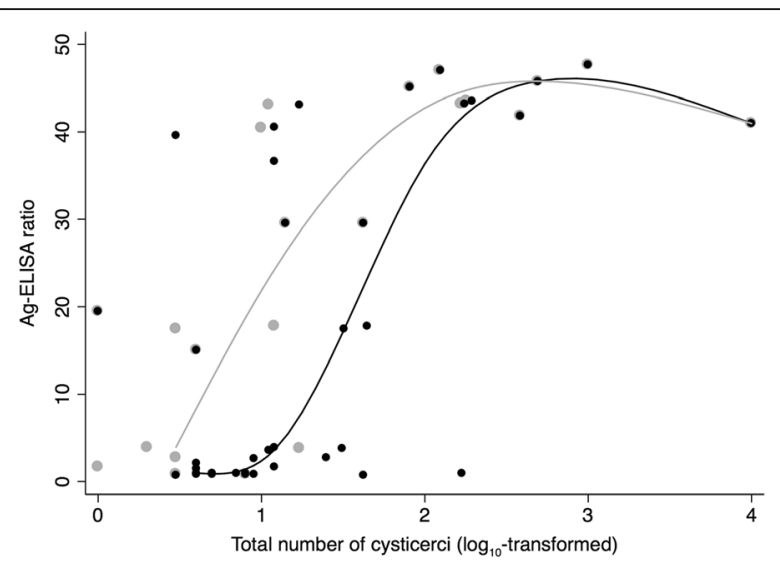

Fig. 2 Relation between Ag-ELISA ratio and the total number of cysticerci $\left(\log _{10}\right.$-transformed; $n=38 \mathrm{~T}$. solium positive animals; indicated in black) and the total number of viable cysticerci $(n=22$ confirmed T. solium infected carcasses with viable cysticerci; indicated in grey). The curves represent the median spline plots, which use the cross medians to fit a cubic spline. Stata/IC 14.1 (StataCorp, College Station, TX, USA)

Product Profile (TPP) for the detection of porcine cysticercosis had been initiated at a WHO stakeholder meeting, and was further finalised after consultation with 53 stakeholders [23]. The TPP indicates the need for a test that at least detects specifically $T$. solium porcine cysticercosis, only viable cysts and preferable in blood spots collected on filter paper. The minimal clinical sensitivity demands were set at $50 \%$ for less than 50 viable cysts and $80 \%$ for more than 50 viable cysts. The minimal clinical specificity was set at $95 \%$. An additional remark was the need for validation in endemic areas to assess crossreactions. Indeed, besides the known fact that the AgELISA used in this study cross-reacts with $T$. hydatigena, false positive results due to exposure to other Taenia spp. were hypothesized [24]. However, in a recent study where piglets were orally infected with Taenia saginata eggs, no specific antibodies (as measured by a commercial western blot) or specific circulating antigens (measured with the B158/B60 Ag-ELISA) could be detected [25], challenging that hypothesis.

An important finding from our study is the presence of T. solium cysticerci in the liver, spleen and lungs, locations that are not routinely included in full carcass dissections. Also, very small (viable) $T$. solium cysticerci were observed in organs and muscles, which could easily be missed, even when slices of $3 \mathrm{~mm}$ are performed. These findings question the true value of full carcass dissection (with the recommended $5 \mathrm{~mm}$ slices) as a gold standard used for the evaluation of interventions and diagnostic tools. Indeed, in our study, this factor may have led to an underestimation of the true specificity of the Ag-ELISA.

The observation of mainly viable cysticerci in the livers of $26 \%$ of the infected carcasses may have important consequences, as in certain cultures, the liver is eaten raw or insufficiently cooked. This finding should be considered in evaluations of control programmes, and also in public health messaging.

\section{Conclusions}

In conclusion, our findings indicate a very high occurrence of porcine cysticercosis in the study area. Full carcass dissection is still recognized as the most sensitive diagnostic tool for the detection of porcine cysticercosis, however dissection of the liver, spleen, kidneys and lungs should be included, and results should be interpreted carefully as small cysticerci can easily be overlooked, leading to false negative results. As an alternative, 'selective dissections' of tongue, diaphragm and masseter or heart muscles could be envisaged, although this does not necessarily lead to better sensitivities than circulating antigen detection. The latter's main drawback is its lack of specificity. The most optimal and most efficient approach will depend on the aims of the diagnosis and might include a combination of both serology and selective dissection of the serologypositives. Other alternatives, such as ultrasound [26], should be further investigated.

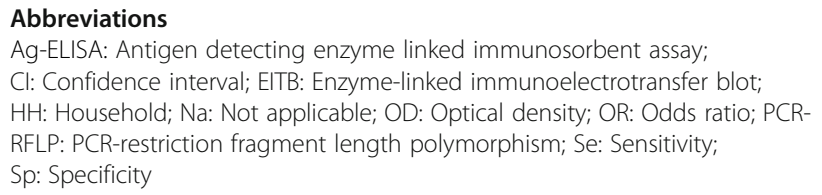

\section{Acknowledgements}

We thank the Ministry of Livestock and Fisheries for the permits to work on animals and for rendering technical support. We thank Mambwe M. Mambo R. Mwango, J. Ndhlovu, J. Kamanga for their technical support, Nyembe and Mtandaza Local communities for their cooperation.

\section{Funding}

The study received financial support from the Institute of Tropical Medicine, Antwerp via the Flemish Ministry of Research. Funding for the PhD study was provided by the Ross University School of Veterinary Medicine. Support was also obtained from the Belgian Cooperation in the framework of the Institutional collaboration between the Institute of Tropical Medicine in Antwerp, Belgium, and the University of Pretoria, Pretoria, South Africa. This publication is also based on research funded in part by the Bill \& Melinda Gates Foundation and with UK aid from the UK Government through GALVmed. The findings and conclusions contained within are those of the authors and do not necessarily reflect positions or policies of the Bill \& Melinda Gates Foundation nor the UK Government.

\section{Availability of data and materials}

The data supporting the conclusions of this article are included within the article. Raw datasets used and/or analysed during the current study are available from the corresponding author on reasonable request.

\section{Authors' contributions}

KEM, IKP, AC, ALW, BD, NS, DB, PD and SG have made substantial contributions to conception and design. CM, KEM, MM, GZ, AVH, AC, PD and SG have made substantial contributions to the acquisition of data. CM, KEM, IVD, BD, NS, DB, PD and SG have made substantial contributions to the analysis and interpretation of data. CM, KEM, IVD and SG have been involved in drafting the manuscript. All authors have been involved in the critical revision of the manuscript for important intellectual content. All authors have participated sufficiently in the work to take public responsibility for 
appropriate portions of the content; and all authors have agreed to be accountable for all aspects of the work in ensuring that questions related to the accuracy or integrity of any part of the work are appropriately investigated and resolved. All authors read and approved the final manuscript.

\section{Ethics approval and consent to participate}

All procedures employed in this study were approved by the University of Zambia Biomedical Research Ethics Committee (004-09-15) and the Ministry of Agriculture and Livestock, Lusaka- Zambia.

\section{Consent for publication}

Not applicable.

\section{Competing interests}

The authors declare that they have no competing interests.

\section{Publisher's Note}

Springer Nature remains neutral with regard to jurisdictional claims in published maps and institutional affiliations.

\section{Author details}

${ }^{1}$ Department of Paraclinical Studies, School of Veterinary Medicine, University of Zambia, P.O. Box 32379, Lusaka, Zambia. ${ }^{2}$ Department of Clinical Studies, School of Veterinary Medicine, University of Zambia, P.O. Box 32379, Lusaka, Zambia. ${ }^{3}$ Faculty of Veterinary Medicine, Ghent University, Salisburylaan 133 , 9820 Merelbeke, Belgium. ${ }^{4}$ One Health Center for Zoonoses and Tropical Veterinary Medicine, Ross University School of Veterinary Medicine, P.O. Box 334, Basseterre, St Kitts, Saint Kitts and Nevis. ${ }^{5}$ Department of Biomedical Sciences, Institute of Tropical Medicine, Kronenburgstraat 25, 2000 Antwerp Belgium. ${ }^{6}$ District Medical Office, Ministry of Health, P.O. Box 30205, Lusaka, Zambia. ${ }^{7}$ Global Alliance for Livestock Veterinary Medicines, P.O. Box 52773 00100, Valley Arcade, Nairobi, Kenya. ${ }^{8}$ Department of Public Health and Surveillance, Scientific Institute of Public Health (WIV-ISP), Rue Juliette Wytsman 14, 1050 Brussels, Belgium. 'Université catholique de Louvain, Institute of Health and Society (IRSS), School of Public Health, 1200 Woluwe-Saint-Lambert, Brussels, Belgium.

Received: 7 August 2017 Accepted: 2 November 2017

Published online: 16 November 2017

\section{References}

1. Murrell KD. In: Murrell KD, editor. WHO/OIE/FAO guidelines for the surveillance, prevention and control of Taeniosis/Cysticercosis. Paris, France: World Health Organization for Animal Health (OIE); 2005. p. 57-71.

2. World Health Organization. Estimates of the global burden of foodborne diseases (FERG), 2015. Geneva: World Health Organization; 2015.

3. Zoli A, Shey-Njila O, Assana E, Nquekam J, Dorny P, Brandt J, Geerts S. Regional status, epidemiology and impact of Taenia solium cysticercosis in western and central Africa. Acta Trop. 2003;87:35-42.

4. Trevisan C, Devleesschauwer B, Schmidt V, Winkler AS, Harrison W, Johansen MV. The societal cost of Taenia solium cysticercosis in Tanzania. Acta Trop. 2017:165:141-54.

5. Sikasunge CS, Phiri IK, Phiri AM, Siziya S, Dorny P, Willingham AL 3rd. The prevalence of porcine cysticercosis in eastern, southern and western provinces of Zambia. Vet J. 2008;176:240-4.

6. Dorny P, Phiri IK, Vercruysse J, Gabriel S, Willingham AL 3rd, Brandt J, et al. A Bayesian approach for estimating values for prevalence and diagnostic test characteristics of porcine cysticercosis. Int J Parasitol. 2004:34:569-76.

7. Mwape KE, Phiri IK, Praet N, Muma JB, Zulu G, Van den Bossche P, et al. Taenia solium infections in a rural area of eastern Zambia - a communitybased study. PLoS Negl Trop Dis. 2012;6:e1594.

8. Mwape KE, Phiri IK, Praet N, Muma JB, Dorny P, Gabriel S. The incidence of human cysticercosis in a rural community of eastern Zambia. PLoS Negl Trop Dis. 2013;7:e2142

9. Mwape KE, Blocher J, Wiefek J, Schmidt K, Dorny P, Praet N, et al. Prevalence of neurocysticercosis in people with epilepsy in the Eastern Province of Zambia. PLoS Negl Trop Dis. 2015:9:e0003972.

10. Gabriel S, Dorny P, Mwape KE, Trevisan C, Braae UC, Magnussen P, et al. Control of Taenia solium taeniasis/cysticercosis: the way forward for subSaharan Africa. Acta Trop. 2016;165:252-60.
11. Phiri IK, Dorny P, Gabriel S, Willingham AL 3rd, Sikasunge C, Siziya S, Vercruysse J. Assessment of routine inspection methods for porcine cysticercosis in Zambian village pigs. J Helminthol. 2006;80:69-72.

12. Sciutto E, MartõÂnez JJ, Villalobos NM, HernaÂndez M, JoseÂA MV, BeltraÂn C, et al. Limitations of current diagnostic procedures for the diagnosis of Taenia solium cysticercosis in rural pigs. Vet Parasitol. 1998;79:299-313.

13. Jayashi CM, Gonzalez AE, Castillo NR, Rodríguez S, García HH, Lightowlers MW. Cysticercosis working Group in Peru. Validity of the enzyme-linked immunoelectrotransfer blot (EITB) for naturally acquired porcine cysticercosis. Vet Parasitol. 2014;199:42-9.

14. Gonzalez AE, Cama V, Gilman RH, Tsang VCW, Pilcher JB, Chavera A, et al. Prevalence and comparison of serological assays, necropsy, and tongue examination for the diagnosis of porcine cysticercosis in Peru. Am J Trop Med Hyg. 1990;43:194-9.

15. Assana E, Kyngdon CT, Gauci CG, Geerts S, Dorny P, De Deken R, et al. Elimination of Taenia solium transmission to pigs in a field trial of the TSOL18 vaccine in Cameroon. Int J Parasitol. 2010:40:515-9.

16. Boa ME, Kassuku AA, Willingham AL 3rd, Keyyu JD, Phiri IK, Nansen P. Distribution and density of cysticerci of Taenia solium by muscle groups and organs in naturally infected local finished pigs in Tanzania. Vet Parasitol. 2002;106:155-64.

17. Sokal RR, Rohlf JF. Biometry: the principals and practice of statistics in biological research. New York: W.H. Freeman; 1981

18. Dermauw V, Ganaba R, Cissé A, Ouedraogo B, Millogo A, Tarnagda Z, et al. Taenia hydatigena in pigs in Burkina Faso: a cross-sectional abattoir study. Vet Parasitol. 2016:30:9-13.

19. Clopper CJ, Pearson ES. The use of confidence or fiducial limits illustrated in the case of the binomial. Biometrika. 1934:26:404-13.

20. Cuzick JA. Wilcoxon-type test for trend. Stat Med. 1985;4:87-90.

21. Brandt J, Geerts S, De Deken R, Kumar V, Ceulemans F, Brys L, Falla NA. Monoclonal antibody based ELISA for the detection of circulating excretory-secretory antigens in Taenia saginata cysticercosis. Int J Parasitol. 1992;22:471-7.

22. Lightowlers MW, Assana E, Jayashi CM, Gauci CG, Donadeu M. Sensitivity of partial carcass dissection for assessment of porcine cysticercosis at necropsy. Int J Parasitol. 2015;45:815-8.

23. Donadeu M, Fahrion AS, Olliaro PL, Abela-Ridder B. Target product profiles for the diagnosis of Taenia solium taeniasis, neurocysticercosis and porcine cysticercosis. PLoS Negl Trop Dis. 2017:11:e0005875.

24. Lightowlers MW, Garcia HH, Gauci CG, Donadeu M, Abela-Ridder B. Monitoring the outcomes of interventions against Taenia solium: options and suggestions. Parasite Immunol. 2016;38:158-69.

25. Dorny P, Dermauw V, Van Hul A, Trevisan C, Gabriël S. Serological diagnosis of Taenia solium in pigs: no measurable circulating antigens and antibody response following exposure to Taenia saginata oncospheres. Vet Parasitol. 2017:245:39-41.

26. Flecker RH, Pray IW, Santivaňez SJ, Ayvar V, Gamboa R, Muro C, et al. Assessing ultrasonography as a diagnostic tool for porcine cysticercosis. PLoS Negl Trop Dis. 2017;11:e0005282.

\section{Submit your next manuscript to BioMed Central and we will help you at every step:}

- We accept pre-submission inquiries

- Our selector tool helps you to find the most relevant journal

- We provide round the clock customer support

- Convenient online submission

- Thorough peer review

- Inclusion in PubMed and all major indexing services

- Maximum visibility for your research

Submit your manuscript at www.biomedcentral.com/submit
Biomed Central 